\title{
Fatores preditivos de coleltíase em obesos mórbidos após gastroplastia em Y de RouX
}

A calculose da vesícula biliar tem íntima relação com a obesidade. Em obesos mórbidos, se considerarmos os indivíduos já colecistectomizados e os que são diagnosticados previamente à cirurgia de gastroplastia, os índices de colecistopatia calculosa chegam a quase $50 \%$ dos pacientes.

Sabe-se que os pacientes submetidos à gastroplastia e que sofrem grande emagrecimento em curto espaço de tempo também estão sujeitos ao aparecimento da colelitíase, principalmente nos primeiros meses do pós-operatório. Alguns autores, na tentativa de prevenir o aparecimento de cálculos biliares após a cirurgia de redução gástrica, usaram o ácido ursodeoxicólico no pós-operatório por seis meses e obtiveram redução no aparecimento de cálculos, porém os resultados não podem recomendar o seu uso rotineiro ${ }^{1,2,3}$.

Devido ao grande número de pacientes que acabam desenvolvendo colelitíase após a gastroplastia, alguns autores estudaram a indicação de colecistectomia profilática por ocasião da cirurgia de redução gástrica. Os resultados se mostraram conflitantes principalmente devido ao surgimento de complicações atribuídas à colecistectomia ${ }^{4,5,6}$.

Os autores do trabalho ora comentado tentam estabelecer fatores preditivos de colelitíase em obesos mórbidos submetidos a gastroplastia. Trata-se de estudo, ao nosso ver, muito importante pois tenta encontrar dados fundamentados estatisticamente para indicação de colecistectomia profilática em vesícula biliar sem cálculos no ato da cirurgia para tratamento da obesidade mórbida.

Os autores estabeleceram como possíveis fatores preditivos na população estudada o peso, o IMC, o colesterol total e frações, e a perda de peso com dois, quatro e seis meses e um ano de pós-operatório.

Os resultados do estudo mostraram que o IMC préoperatório foi um fator preditivo, sendo que os pacientes com valores médios acima de 50 foram os que mais apresentaram cálculos biliares no pós-operatório.

Os níveis de colesterol total e a relação entre colesterol total e a fração HDL-colesterol (fator utilizado para determinar riscos de doenças cardiovasculares) foram significativamente superiores nos pacientes com colelitíase quando comparados aos pacientes que não desenvolveram cálculos.

Também nesse estudo, os níveis de LDL e VLDL colesterol, além dos triglicérides, foram fatores preditivos de colelitíase após gastroplastia em Y de Roux realizada em obesos mórbidos.

Acreditamos que o estudo de Taha et al. trouxe grande contribuição ao relatar fatores preditivos de colelitíase em obesos mórbidos, facilitando assim a indicação de colecistectomia em pacientes alitiásicos durante a realização da gastroplastia.

Cabe lembrar, no entanto, que a dificuldade técnica na realização da colecistectomia em obesos pela via aberta, assim como as complicações inerentes à própria cirurgia podem levar ao aumento da morbi-mortalidade do procedimento combinado. Portanto, sugerimos que o cirurgião que optar pela colecistectomia durante a gastroplastia com vesícula biliar alitiásica, usando os fatores preditivos ora estudados, deverá discutir detalhadamente o procedimento com o seu paciente, colocando claramente os riscos e as possíveis complicações para evitar futuros problemas éticos e legais.

Elias JiRjoss llias

\section{Referências}

I. Villegas L, Schneider B, Provost D, Chang C, Scott D, Sims T, et al. Is routine cholecystectomy required during laparoscopic gastric bypass?. Obes Surg. 2004; I $4(1): 60-6$

2.Caruana JA, McCabe MN, Smith AD, Camara DS, Mercer MA, Gillespie JA. Incidence of symptomatic gallstones after gastric bypass: is prophylactic treatmwent really necessary? Surg Obes Relat Dis. 2005; I (6):564-7.

3.Mason EE, Renquist KE. Gallbladder management in obesity surgery. Obes Surg. 2002; I 2(2):222-9.

4. Taylor J, Leitman IM, Horowitz M. Is routine cholecystectomy necessary at the time of Roux en Y gastric bypass?. Obes Surg. 2006; I 6(6):759-6I.

5.Fobi M, Lee H, Igwe D, Felahy B, James E, Stanczyc M, et al. Prophylatic cholecystectomy with gastric bypass operation: incidence of gallblader disease. Obes Surg. 2002; I 2(3):350-3

6.Liem RK, Niloff PH. Prophylatic cholecystectomy with open gastric bypass operation. Obes Surg. 2004; I 4(6):763-5.

7.Taha MIA, Malheiros CA, Freitas Jr WR, Puglia CR, Lacombe A. Fatores preditivos de colelitíase em obesos mórbidos após gastroplastia em Y de Roux. Ver Assoc Med Bras 2006; 52(6): 430-4. 